\title{
Accelerated orthodontic treatment by alveolar corticotomies: a clinical and experimental approach*
}

\author{
Marie-Céline Sitbon and Jean-Baptiste Charrier
}

Directeur de mémoire : Christophe DUNGLAS / Paris V

ABSTRACT

Indications for orthodontic treatments are in constant augmentation in the adult population. Patients want the shortest possible treatments. Alveolar corticotomies speed up the tooth movement in comparison to conventional orthodontic treatments, therefore decrease treatment duration.

This technique consists in the surgical section of cortical bone that leaves the medullar bone uninjured. The biological effects of corticotomies rely on decreasing medullary bone density and increasing bone remodelling activity (RAP regional acceleratory phenomenon).

We are currently testing this combined approach of alveolar corticotomies and orthodontic treatments on a pig model with special attention given to potential secondary effects on pulp vitality, dental root morphology and length.

We also present here two adult patients with malocclusion cases who underwent alveolar corticotomies as part of their surgical-orthodontic treatments.

These experimental and clinical preliminary studies confirm that corticotomy requires a shorter time of orthodontic movement. Our clinical results showed that this technique resolves malocclusion at least twice as fast as conventional orthodontic treatment. observed.

No secondary effects of corticotomies on pulp vitality dental root morphology were

\section{KEYWORDS}

Alveolar corticotomy

Malocclusion

Orthognatic surgery 
Address for correspondence:

M.-C. SITBON,

136, boulevard Saint-Denis,

92400 Courbevoie.

mcelinesitbon@gmail.com

J.-B. CHARRIER,

$M D, P h D$, Service de chirurgie de la face et du cou,

CHU de Bicêtre, AP-HP,

Université Paris Sud-11,

78, avenue du Général Leclerc,

94275 Kremlin Bicêtre Cedex, France.

* The English version of this paper is not available; to read the French version, go to http://odf.edpsciences.org/10.1051/odf/2009005 